Revue de l'Institut des langues et cultures

d'Europe, Amérique, Afrique, Asie et Australie

$16 \mid 2012$

La culture progressiste à l'époque de la guerre froide

\title{
Die sozialistische Armee als eine Kulturmacht? Die Tschechoslowakische Armee 1950-1956
}

The Socialist Army as a Cultural Power? The Czechoslovak Army 1950-1956

\section{Václav Šmidrkal}

\section{OpenEdition}

Journals

Édition électronique

URL : http://journals.openedition.org/ilcea/1277

DOI : $10.4000 /$ ilcea. 1277

ISSN : 2101-0609

\section{Éditeur}

UGA Éditions/Université Grenoble Alpes

\section{Édition imprimée}

ISBN : 978-2-84310-232-5

ISSN : $1639-6073$

Référence électronique

Václav Šmidrkal, „Die sozialistische Armee als eine Kulturmacht? Die Tschechoslowakische Armee 1950-1956", ILCEA [Online], 16 | 2012, Online erschienen am: 04 Juli 2012, abgerufen am 22 März 2021. URL: http://journals.openedition.org/ilcea/1277 ; DOI: https://doi.org/10.4000/ilcea.1277

Ce document a été généré automatiquement le 22 mars 2021

(C) ILCEA 


\title{
Die sozialistische Armee als eine Kulturmacht? Die Tschechoslowakische Armee 1950-1956
}

\author{
The Socialist Army as a Cultural Power? The Czechoslovak Army 1950-1956
}

\author{
Václav Šmidrkal
}

\section{Die „Fortschrittlichkeit“ der sozialistischen Kultur}

1 Die Idee des gesellschaftlichen Fortschritts, die ideologisch auf dem historischen Materialismus Karl Marx' aufbaute, wurde zum Aushängeschild der kommunistischen Bewegung. Dieser behauptete, Sozialismus und Kommunismus seien laut Naturgesetzlichkeit des historischen Materialismus die höchsten Entwicklungsphasen der menschlichen Gesellschaftsordnung. Während im Sozialismus ,jeder nach seinen Fähigkeiten“ arbeite, und „nach seiner Leistung“ belohnt werde, berechne sich im Kommunismus die Belohnung nach dem "jeweiligen Bedürfnis“. Damit werde die geschichtliche Entwicklung der Menschheit zu Ende gebracht.

Der Fortschritt wurde von den Kommunisten gern als ein synonymisch gebrauchter Tarnname für Sozialismus und Kommunismus benutzt, da die allgemeine Idee des Fortschritts „sicherer und angenehmer" erschien als die rot gefärbten Vorstellungen der Zukunft (Hayduk, 2008, S. 238). Ohne ideologische Voraussetzungen erwartete man vom Fortschritt, dass „materielle, politische, intellektuelle und moralische Bedingungen sich fortlaufend und unumgänglich im Laufe der Menschheitsgeschichte verbessern und dass so eine Verbesserung auch in der absehbare Zukunft andauern wird" (Weiler, 2007, S. 3669). Obwohl die Fortschrittstheorien in der Soziologie schon seit Max Weber als "unhaltbar“ abgelehnt werden, bleiben sie im linksradikalen Denken trotz des Kettenuntergangs der kommunistischen Diktaturen in Ostmitteleuropa Ende der achtziger Jahre bis heute anwesend. 
3 Ein Teil von den revolutionären Umwälzungen in der Gesellschaft, die die kommunistischen Parteien anstrebten, war auch die „kulturelle Revolution“. Ihr Ziel war es, den „sozialistischen Menschen“ zu schaffen, der sich sozialistische Denkensund Verhaltensmuster angeeignet hat. Ohne die geistigen Umwandlungen konnten nach deren Vorstellung die revolutionären Fernziele nicht erreicht werden. Die Kultur hatte dabei eine entscheidende Rolle zu spielen. Ihre angebliche Stärke bestand in der Anknüpfung an die marxistisch-leninistische Ideologie, die eine fortschrittliche gesellschaftliche Ordnung „wissenschaftlich“ garantierte. Laut Marxismus-Leninismus war Kultur im Kapitalismus von Klassengegensätzen geprägt und jede Klasse lebte in ihrer eigenen Kultur. Die Hierarchie der „Kulturen“ sah man als von der herrschenden Klasse bestimmt: Die Bourgeoisie selber genieße eine Auslese des Kulturguts und schreibe dem Proletariat eine erdrückende Massenkultur vor. Die sozialistische Kultur versprach dagegen eine Überwindung der klassenmäßigen Teilung der Kultur. Die „Demokratisierung“ im Sinne der Erweiterung des Kulturangebotes für alle und der Entfaltung der schöpferischen Kräfte des bis jetzt unterdrückten Volkes gehörten zu den deklarierten Hauptaufgaben der Kulturpolitik im Staatssozialismus (Hanke, 1986, S. 233).

4 Für den Bereich der Kunst wurde nach dem Zweiten Weltkrieg der sozialistische Realismus als eine verbindliche Kunstdoktrin aus der Sowjetunion in die Staaten Ostmitteleuropas in der Ždanov'schen Ausprägung importiert. Volksverbundenheit (narodnost'), Parteilichkeit (partiinost') und Klassengemäßheit (klassovost') waren die drei Grundsätze, wonach die Kunstwerke beurteilt wurden. Die unklare Auslegung dieser Grundsätze, die offensichtliche Dienstbarkeit solcher Kunstwerke und die wenig überzeugenden künstlerischen Ergebnisse diskreditierten jedoch nach und nach den sozialistischen Realismus.

5 In Rückblende scheint für die sozialistische Kulturpolitik die Methode von Zuckerbrot und Peitsche charakteristisch. Durch die zentral geplante Ökonomik und die großzügigen staatlichen Zuschüsse baute der Staatssozialismus zwar manche existenziellen Schwierigkeiten der Kulturbetriebe und soziale Unsicherheiten der Künstler ab. Dafür verlangte er jedoch von den Künstlern, dass sie die Steuerungsfunktion der Partei anerkannten und sich selbst sowie ihre Kunst den politischen Forderungen unterwarfen.

$6 \quad$ Hand in Hand mit der staatlichen Förderung und Lenkung der Kunst und Kulturarbeit erklärte das Regime der „dekadenten bürgerlichen Kultur“ einen Krieg und im weiteren Sinne allen sonstigen „schädlichen“ Kultureinflüssen. Am Pranger stand vor allem die westliche populäre Kultur, die zum Zorn der kommunistischen Funktionäre trotz Verboten, Beschlagnahmen und Strafen langfristig einen guten Zuspruch bei den sozialistischen Bürgern fand.

\section{Armee und Kunst: Die Kunst als Waffe}

7 Die sozialistische Armee wurde von den offiziellen östlichen Theoretikern als ein höherer, fortschrittlicher Entwicklungsgrad der Streitkräfte gelobt. Ihr Klassencharakter, der den Belangen des Volkes entspreche, und die angeblich friedliche Absichten gehörten für sie zu ihren Vorzügen gegenüber der kapitalistischen Militärformationen (Wiatr, 1964, S. 283 ff.). Darüber hinaus stilisierte sich die sozialistische Armee, während eine kapitalistische Armee stereotypisch als eine 
Institution betrachtete, die die kulturellen Werte zerstöre und die Menschenwürde missachte, in die Rolle eines bewaffneten Organs, das die kulturellen Werte achte, schütze und sogar selbst schaffe. Auch dadurch sollte ihre Überlegenheit gegenüber den kapitalistischen Streitkräften demonstriert wurden.

„Kunst ist Waffe“, lautete die später oft wiederholte kommunistische Losung, die einem Gedicht von Friedrich Wolf (1928) entstammte. Die Vermutung einer mobilisierenden Kraft, die sich in den Kunstwerken verbergen kann, wurde im sozialistischen Armeemilieu weiter konkretisiert. Von der Kunst wurde unverblümt ein Beitrag zur Erhöhung der Kampfmoral gefordert. So entstand ein langfristiger Konflikt zwischen der engagierten, politisch-kämpferischen Kunst, die von den politischen Offizieren gern gesehen wurde, und der leichten, meist unpolitischen Unterhaltungskunst, die die vielen Armeeangehörigen bevorzugten.

In den sozialistischen Streitkräften gingen allerdings ähnliche kulturpolitische Prozesse wie im Zivilbereich vor. Die Kulturpolitik der sozialistischen Armee verfolgte demzufolge zwei Ziele: erstens die Hebung des allgemeinen Kulturniveaus durch ein vielseitiges Kulturangebot und Einbeziehung von Laienkünstlern aus den Reihen der Armeeangehörigen in die regelmäßige künstlerische Tätigkeit, und zweitens den Aufbau der hauptamtlichen kulturellen Institutionen, die für die kulturelle Betreuung der Truppen, die methodische Anleitung der schaffenden Soldaten und die Repräsentation der Armee vor der Öffentlichkeit zuständig waren. Die militärische Abart des „sozialistischen Menschen“ hieß die „sozialistische Soldatenpersönlichkeiten": eine ausgewogen gebildete Persönlichkeit mit tiefer politischer Überzeugung und einem hohem Grad an Kampfbereitschaft.

In der Armee wurde eine Art Kohledurchschlag der zivilen organisatorischen Maßnahmen eingeführt. An Stelle von Kulturhäusern baute man „Häuser der Armee“ auf, an Stelle von Betriebsklubs schaffte man „Politische Aufklärungszimmer" und „Klubs“. Anders als im Zivilbereich, wo der Hauptlast der Organisierung bei den Gewerkschaften lag, wurden diese Aufgaben von den stellenmäßigen „Kulturoffizieren“ und ehrenamtlichen Funktionären aus den Reihen der Soldaten übernommen. Neben der Betätigung von Laienkünstlern entstanden auch neue hauptamtliche Kunsteinheiten, die in die Militärstruktur eingebaut wurden. Die mannigfaltigen militärischen Kunstensembles wurden zum Flaggschiff der jeweiligen Armeekultur (Piekarski, 2002; Rogg, 2008, S. 167-169; Šmidrkal, 2010).

\section{Kultur als Beweis des Fortschritts?}

11 Im Höhepunkt des kulturellen Aufbaus in der Tschechoslowakischen Armee 1953 gehörten zum Netz der professionellen kulturellen Einrichtungen drei ständige Schauspielbühnen (Theater der Tschechoslowakischen Armee, Armeekunsttheater in Prag und Armeetheater in Martin in der Slowakei), vier hauptamtliche Kunstensembles (Zentrales, je eins beim 1. und beim 2. Militärbezirk und eins bei der Luftwaffe), eine Oper (nur 1952-1955), ein Studio der bildenden Künste, ein großes Verlagskombinat, das unter vielen anderen Erzeugnisse auch eine militärische Tageszeitung herausgab, ein Filmstudio und Filmdistribution (1954 umfasste das Netz fast 550 Armeekinosäle), eine Rundfunkredaktion, ein breites Netz von Häusern der Armee, Klubs und Militärbibliotheken, ein zentrales Orchester und 24 andere Militärmusikkorps (1952), ein Militärmuseum und andere unterstützende Einrichtungen (Šach, 2006, S. 179-182). 
Die Armeeangehörigen betätigten sich massiv in der Zirkelarbeit, gründeten Laienkunstgruppen, nahmen an vielen kulturellen Wettbewerben und Kampagnen teil. Die „kulturelle Aufklärungsarbeit“ (kulturně osvětová práce) wurde als ein Unterkapitel der „politischen Massenarbeit“ systematisch aufgebaut.

Mitte 1950 waren jedoch die Institutionsgefüge sowie die Ausbreitung der Laienbetätigung und der kulturellen Betreuung noch relativ bescheiden. Sie konnte sich auf keine eigene bedeutendere Tradition wie beispielsweise in Polen stützen und musste deshalb fast vom Anfang an aufgebaut werden (Piekarski, 1999; Wyszczelski, 2004). Obwohl die kommunistische Machtübernahme 1948 als eine tiefgreifende Zäsur der tschechoslowakischen Geschichte gilt, wurde die Kulturpolitik der Armee erst zwei Jahre später weitreichend umgewandelt. Im April 1950 wurde der Minister für Nationale Verteidigung, Armeegeneral und Held beider Weltkriege, Ludvík Svoboda (1895-1979), aus seinem Amt enthoben und durch den stalinistischen Karrieristen Alexej Čepička (1910-1990) ersetzt. Der Vier-Sterne-General Čepička hatte nicht einmal den Grundwehrdienst abgeleistet und wurde so plötzlich vom Soldaten zum Armeegeneral und zweithöchsten Mann in der Armeehierarchie befördert (nach seinem Schwiegervater und Präsidenten der Republik Klement Gottwald, der nominell der Oberbefehlshaber der Armee war). Čepička hatte zwar keine militärische Ausbildung und nicht einmal ein Fingerspitzengefühl für militärische Angelegenheiten, verfügte aber über Ehrgeiz, die Armee - im Einklang mit den Forderungen Stalins - so groß und stark wie möglich aufzubauen. Von dieser Machtposition aus konnte er schließlich auch seine langjährige Vorliebe für Theater und Kunst $\mathrm{zu}$ seinem politischen „Programm“ machen (Pernes, Pospíšil, Lukáš, 2008, S. 21-25, 60-61). Es waren daher vor allem seine persönliche Neigung und seine politischen Möglichkeiten, die zum Aufbau des überdimensionalen Kultursystems führten.

Die Kompromisse der „dritten Republik“ (1945-1948) in Sachen der Kulturarbeit in der Armee wurden endgültig zugunsten der Kommunisten überwunden. Die Aufklärungsoffiziere (osvětoví důstojníci), die 1945 nach dem Vorbild der sowjetischen politischen Kommissare eingesetzt wurden und die im Unterschied zur Roten Armee zwar politisch, aber unparteilich Soldaten erziehen sollten, wurde spätestens 1950 durch den Politapparat ersetzt. An der Spitze stand die Politische Hauptverwaltung, die einem Ausschuss des Zentralkomitees der kommunistischen Partei gleichkam. Dagegen mussten die Militärgeistlichen, die in einem beschränkten Maße 1945 zugelassen worden waren und theoretisch ein Gegengewicht gegenüber den Politoffiziere sein konnten, 1950 die Armee verlassen, und der „wissenschaftliche Atheismus“ wurde stark forciert.

14 Einer der geduldeten Kompromisse der dritten Republik war das Weiterbestehen des Armeekunstensembles „Vít Nejedlý“, das an der Ostfront 1942 als das tschechoslowakische Musikkorps unter der Leitung von Vít Nejedlý (1912-1945) gegründet wurde. Das kleine Ensemble, das sich überwiegend aus Laienmusikern in Uniform zusammensetzte, erfüllte nach innen die Aufgaben der kulturellen Betreuung der eigenen Truppen und nach außen die Repräsentation der auf der Seite der Alliierten kämpfenden Tschechoslowaken. Bis sie im Mai 1945 nach Prag kamen, erweiterte sich das Musikkorps in ein Militärkunstensemble. Im Frieden entstand jedoch die Frage, ob dieses Ensemble, obwohl es im Krieg gut gearbeitet hatte, nicht demobilisiert (d.h. aufgelöst) werden sollte. Die Befürworter des Weiterbestehens argumentierten mit der Notwendigkeit der Truppenbetreuung und der vielseitigen 
Wehrerziehung der Zivilbevölkerung. Die Kritiker fürchteten sich vor den finanziellen Ausgaben, dem faktischen Abzug der Wehrpflichtigen, die in diesem Ensemble den Grundwehrdienst ableisten würden, und der mangelhaften künstlerischen Qualität der Produktion im Vergleich zu den traditionellen Institutionen, die ihre Arbeit im Frieden wieder entfalten konnten. Es waren die Befürworter, die sich durchsetzten, und das Ensemble blieb in die militärischen Stellenpläne eingegliedert.

15 Anfang der fünfziger Jahre wurde das Ensemble als Armeekunstensemble „Vít Nejedlý“ (AUS VN) professionalisiert und stellenmäßig erheblich erweitert (bis zu 250 Arbeitsstellen). Damit begann die Aufbauwelle der Ensembles. Bei dem 2. Militärbezirk (geographisch im Osten der Republik) stellte eine Laiengruppe den Antrag auf Professionalisierung und wurde 1951 als Militärkunstensemble "Ján Nálepka“, das sich vorwiegend mit der slowakischen Kultur beschäftigt, aufgenommen. Kurz danach folgte der 1. Militärbezirk (im westlichen Teil der Republik) mit der Forderung, dass, wenn der 2. Militärbezirk ein Ensemble habe, auch beim 1. Militärbezirk ein Pendent vorgesehen werden sollte, das sich für den Einsatz in der ersten strategischen Staffel vorbereiten werde. Schließlich meldete sich auch die Luftwaffe, dass in ihr als einer besonderen Waffengattung auch ein eigenes Ensemble notwendig sei. Dieser „Schneeballeffekt" sagt als auch etwas über die damals bestehende Begeisterung und Eigeninitiative der Basis sowie über die unklaren Vorstellungen der Armeeführung über die Ziele des Kultursystems aus.

Das Programmangebot der Ensembles setzte sich in den Anfangsjahren aus stark politisierten Vaudevilles, Estraden oder auch gewöhnlichen Konzerten zusammen. Die Programme bestanden aus „Nummern“ (Tänze, Lieder, Kompositionen, Rezitationen, Sketches), die manchmal durch Begleitworte verbunden wurden. Die Produktion der Ensembles war jedoch gewissermaßen ähnlich und austauschbar.

Nichtsdestoweniger wurden die Ensembles zum Signet der militärischen Kulturpolitik dieser Jahre und wurden auch oft im Ausland eingesetzt. Für das AUS VN gehörte seine viermonatige Tournee durch China 1952 zu den größten Ereignissen. Das VUS JN triumphierte in ähnlicher Weise 1954 in Bulgarien. Auch die Tschechoslowakei empfing die Ensembles der sozialistischen Armee mit großem Pomp. Das Alexandrow-Ensemble wurde 1950 und 1953 stürmisch von der tschechoslowakischen Bevölkerung begrüßt. Ebenso führten die Ensembles der chinesischen Befreiungsarmee und der nordkoreanischen Armee erfolgreiche Tourneen quer durch die ganze Tschechoslowakei durch.

$\mathrm{Zu}$ den Symbolen der Macht und des Prestiges der Armee gehörte vor allem das traditionsreiche Theater "Na Vinohradech“, das 1907 als Theater des tschechischen Bürgertums eröffnet wurde. Die erste Saison des Theater als „Theater der Tschechoslowakischen Armee“ wurde am 9.Oktober 1950 („Tag der Armee“ in Erinnerung an die Kämpfe im Dukla-Pass 1944 und die Überschreitung der ehemaligen tschechoslowakischen Grenze) mit dem Stück „Dukla“ über dieses geschichtliche Ereignis uraufgeführt. Das durch ein Übermaß an Pathos und Unglaubwürdigkeit gekennzeichnete Stück wurde durch das Publikum relativ gut aufgenommen. Was von höchsten militärpolitischen Kreisen als erster Erfolg auf dem richtigen Weg belobigt wurde, war eine grobe Verklärung der Geschichte unter Einsatz von schwachen künstlerischen Mitteln. Der Held der Sowjetunion, Generalmajor Richard Tesařík (1915-1967), der beim Dukla-Pass persönlich mitgekämpft hatte und gegenüber dem Minister Čepička kritisch eingestellt war, lud nach der Uraufführung einige 
Schauspieler zu sich ein, wo er über die Tragödie der Dukla-Operation erzählte, die viele menschliche Leben sinnlos gekostet hatte (Bezouška, 1993, S. 222). Dieser Zwiespalt zwischen der mediatisierten und der erlebten Realität wurde in der ersten Hälfte der fünfziger Jahre fast zur Regel.

Von der kulturellen Aufklärungsarbeit wurde seitens der Politorgane mehr als bloße Entspannung und Erholung der Soldaten erwartet. Sie sollte erziehen und zur Kampfbereitschaft durch Erhöhung der politisch-ideologischen Motivation und Moral der Truppen beitragen. Es wurde an einen Spill-over-Effekt geglaubt, das vorsah, dass gute Ergebnisse in der Kulturarbeit sich positiv auch auf dem Gebiet der militärischen Ausbildung widerspiegeln würden. Obwohl die kulturelle Aufklärungsarbeit politisiert und oft nur formal durchgeführt wurde, hatte sie eine positive Bedeutung auch für die Soldaten. Die zweijährige Wehrpflicht (manchmal bis drei Jahre verlängert) an einem von dem Zuhause entfernten Ort und mit einer geringen Zahl von Ausgängen oder Urlaubstagen zwang die Soldaten zu Freizeitgestaltung innerhalb der Kasernen. „Kultur“ wurde schnell als eine Möglichkeit zur Erleichterung des Dienstes und auch zum Erwerb von Privilegien entdeckt. Die Mitwirkenden opferten zwar ihre Freizeit für die Proben, aber wenn es Auftritte gab, konnten sie sogar von den Dienstverpflichtungen befreit werden, da die Kommandantur auch auf die Kulturarbeit Rücksicht nahm.

Neben den verschiedensten Jahrestagen des kommunistischen Kalenders wirkten auch künstlerische Wettbewerbe und Leistungsvergleiche motivierend. Die bürokratisch vorgeschriebenen Teilnehmerzahlen und demzufolge die gezwungene Teilnahme der Soldaten führte oft $\mathrm{zu}$ einem unausweichlichen Formalismus der Arbeit. 1953 arbeiteten in den politischen Aufklärungszimmern und den Klubs mehr als 1.450 Laiengruppen mit circa 39.000 Mitgliedern (Šach, 2006, S. 177). Unrühmlich bekannt war der Leserwettbewerb um das Fučík-Leserabzeichen, eine Massenkampagne unter den Jugendlichen, die nach dem kommunistischen Journalisten und Widerstandskämpfer Julius Fuč́́k (1903-1943) benannt worden war. Die Kandidaten sollten vor einer Prüfungskommission eine gute Kenntnis von einigen politisch wertvollen literarischen Werken nachweisen, um ein Abzeichen zu bekommen. Der Druck zur Massenbeteiligung (in den Jahren 1951-1953 wurden mit dem Abzeichen rund 56.000 Soldaten ausgezeichnet) und zum Studium der unpopulären politischen Literatur (die Auswahl der Werke war sehr begrenzt, in der Pflichtlektüre waren z. B. einigen Schriften Lenins, Stalins, Kalinins und Gottwalds) führte zur Entwertung des Abzeichens und der Wettbewerb wurde später eingestellt (Bílek, 1987, S. 92).

\section{6 - ein unauffälliger Wendepunkt}

Das unangemessen große, finanziell aufwendige und uneffektive Kultursystem sowie die künstlerische Unfreiheit und Lenkung der Kultur durch politisch-militärische Befehle wurden vom Teil der Basis früh als falsch empfunden; dies konnte aber im Rahmen der starken Repressalien der frühen fünfziger Jahren nur sehr vorsichtig artikuliert werden. Als ein Vorspiel für das Jahr 1956 und die Enthüllungen des 20. Parteitages der KPdSU über die "Deformationen“ des Stalinismus galt in der Armeekultur das Theaterspiel Pavel Kohouts mit dem Titel "Septembernächte“ (Záríijové noci). Der einst eifrige kommunistische Funktionar und der Partei ergebener Autor schrieb anhand seiner erschütternden Erfahrung aus seiner kurzen Dienstzeit bei 
einem Truppenteil ein Theaterstück über das gegenwärtige Armeeleben, wo er die Mängel offen kritisierte. Das Stück wurde Ende 1955 an der Bühne des Theaters der Tschechoslowakischen Armee in Prag uraufgeführt. Zuerst kam es relativ positiv an und wurde mehrmals gezeigt, bis es überraschenderweise als eine unzulässige Kritik verboten und der Autor zum Ausschluss aus der Partei vorgeschlagen wurde. Einige Wochen später begann der 20. Parteitag der KPdSU, dessen Ergebnisse ein neues Licht auch auf das Theaterspiel „Septembernächte“ warfen. Plötzlich wurde es als ein wertvolles Beispiel der notwendigen Kritik begrüßt und zur Inszenierung empfohlen. Im folgenden Jahr wurde nach dem Spiel ein gleichnamiger Film gedreht. Obwohl die Kritik dieses Werkes sich nur gegen die unfähigen Offiziere richtete (die Arbeiter, die in einer Werbekampagne direkt aus den Betrieben in die Armee als offiziere aufgenommen wurden) und die in dem Stück dargestellten Probleme wurden per deus ex machina gelöst, war es ein wichtiger Schritt auf dem Weg zur offiziellen regimekritischen Literatur. Ohne die bisher verbindliche rosa Brille zeigte es die Armee und einige von ihren Soldaten als Menschen, deren Zynismus und Gleichgültigkeit durch die bestehenden Bedingungen in der Volksarmee verursacht wurden.

Eine Entstalinisierung wie in Polen oder in Ungarn gab es in der Tschechoslowakei nicht (Blaive, 2005). Obwohl es viele kritische Stimmen gegenüber der bisherigen Praxis gab, blieb die Tschechoslowakei als eine Bastion des Konservatismus zwischen den zwei Ländern, deren Bevölkerung sich in Bewegung setzte. Eine der wenigen Veränderungen war die Absetzung des mächtigen Ministers Čepička im April 1956. Er galt als „Prügelknabe“ und seine Amtszeit wurde nicht nur in den heimlichen Tagungen des Politbüros des Zentralkomitees, sondern auch in der Grundorganisation der Partei scharf kritisiert.

Vor dem Zentralkomitee versuchte Čepička seine als „größenwahnsinnig“ kritisierte Kulturpolitik noch rückwärts - und vergeblich - zu rechtfertigen:

Ich glaubte, dass in unserem Land, das zehn professionelle Ensembles und Orchester, Theater und zwölf Opern hatte, womit im Verhältnis zur Bevölkerungsstärke sich kein anderes Land auf der Welt brüsten konnte, es keine übertrieben Forderung wäre, dass die Armee neben dem Zentralkunstensemble und dem Zentraltheater noch in jedem Bezirk ein Ensemble und ein Theater hatte. In der Errichtung der Armeeoper sah ich den Vorteil und die Überlegenheit unserer volksdemokratischen Ordnung, die auch den Soldaten in entfernten Garnisonen und in den Sommerlagern diesen Spitzenausdruck der Kunst zugänglich machen konnte. [...] Die Praxis hat gezeigt, dass diese Ansichten falsch waren, die realen Bedürfnisse der Armee in der Praxis übertroffen wurden und dass es sogar zu einer gewissen Isolierung von dem kulturellen Leben des Landes kam. (Kaplan, 2011, S. 197)

24 Nicht nur das Ende dieses Ministers, sondern auch eine gewisse Entspannung der internationalen Lage nach dem Viermächtetreffen in Genf 1955 hatte Einfluss auf die Veränderung der militärischen Kulturpolitik. Die Armee bekam den Auftrag, die Stellenpläne in Richtung einer Verkleinerung zu überarbeiten. Bis 1960 wurden die Theater an die Zivilverwaltung zurückgegeben. Das Ensemble der Luftwaffe „Vítězná kř́́dla“ wurde aufgelöst und der überwiegende Teil davon ins Prager Theater „Rokoko“ übernommen, wo die bewährten Künstler ihre Karriere fortsetzen können. Es wurde auch über das weitere Schicksal der verbleibenden militärischen Kulturinstitutionen diskutiert, aber radikale Vorschläge für das Loswerden der Last der militärischen Kulturinstitutionen kamen nicht durch. 


\section{Schlussbemerkungen}

In der kulturpolitischen Praxis der Tschechoslowakischen Armee der Jahre 1950-1956 wurden die kommunistischen Ideale der fortschrittlichen Kultur nur teilweise erfüllt: Die Theorie und die Praxis klafften auseinander. Noch stärker als im bürgerlichen Kapitalismus wurde der Gegensatz zwischen den Mächtigen, die die Kultur prägen, und den Ohnmächtigen, die sich an das, was vorgeschrieben wurde, halten mussten, deutlich. Die politische Elite ließ sich selber und ihre Politik allseits verherrlichen und die Kunst diente ihr als ein Instrument der symbolischen Kommunikation, das zur Steuerung der Gesellschaft bestimmt war. Diese „Abweichung“ wurde nach 1956 verharmlosend als „Kult der Persönlichkeit“ bezeichnet und vorsichtig abgelehnt. Die Konzeption der Kulturarbeit stand nichtsdestoweniger auf einer Kreuzung. Sie musste „abnehmen“, das Gute und Bewährte sollte aber nicht über Bord geworfen werden.

Von den professionellen Künstlern wurde zwar die Entlastung von dem Druck der Macht, die ihnen das Engagement in dem militärischen Kultursystem ermöglichte, als positiv bewertet. Die Künstler erlangten dadurch mehr finanzielle Freiheit, die aber gleichzeitig wieder auf andere Weise unterbunden wurde. Straffe politische Lenkung und Repressalien bedrohten alle Andersdenkenden. Das militärische Segment der sozialistischen Kultur war per se mehr mit der Partei verbunden als die Kultur anderswo, da die ganze Armee eine „Parteiarmee“ war und Kultur der Politarbeit unterstellt. Ein anderes Problem bestand darin, dass die erziehende Kunst des sozialistischen Realismus nicht nur wegen ihrer Schablonenhaftigkeit und ihres Formalismus in eine Sackgasse geriet. Der ihr offiziell zugeschriebene fortschrittliche Charakter stand im Widerspruch mit ihren antimodernen Erscheinungsformen.

Am erfolgreichsten waren die kulturellen Aktivitäten als Freizeitangebot für die Soldaten. Sie kamen oft zum ersten Mal in Kontakt mit dem Theater, dem Film, schöngeistiger Literatur, bildenden Künsten. Kultur half ihnen oft, die schwierige Zeit „bei der Fahne“ zu überleben. Dabei darf man aber nicht vergessen, dass viele Armeeangehörige die Armee von der Schattenseite her kennenlernten: als Soldaten in den Strafbataillonen für politisch Unzuverlässige. Auch dort wurde aber manchmal die Kulturarbeit als eine Erziehungsmaßnahme durchgeführt.

Nach Ablösung Čepičkas und im Zuge der Senkung der Personalstärke der Armee stellte man sich die Frage, wie viel aus der Erbschaft Čepičkas im Kulturbereich abgebaut sollte und wie man den Rest optimal umwandeln könne. Das System der kulturellen Einrichtung wurde einerseits reduziert, andererseits befanden sich aber die restlichen Institutionen in einer langjährigen Identitätskrise. Mehr reformfähig waren die Einrichtungen, die nicht aus einer sogenannten ruhmreichen Vergangenheit nutznießen könnten. Die privilegierte Stellung des AUS VN erwies sich als eine Bremse auf dem Weg zur Reform.

Dieses kommunistische Experiment, das von der Durchsetzung des Stalinismus und den Umständen eines Höhepunktes des Kalten Krieges eingerahmt wurde, zeigte, dass die sozialistische Armee tatsächlich als eine - wenn auch eher despotische - kulturmacht wirken konnte. Es geschah allerdings unter einem starken Einsatz von staatlicher Gewalt. Ohne Zwang hätte die Armee nicht so viele prächtige Künstler für ihre Aufträge gewonnen, und auch die Beteiligung der Soldaten wäre niedriger gewesen. Immerhin 
nahmen viele von ihnen aus eigener Motivation und daher zwangslos am Kultursystem der sozialistischen Armee teil.

\section{BIBLIOGRAPHIE}

BEZoUšKA Bohumil, Jakjsem proskotačil život [Wie ich mich durch das Leben getummelt habe], Prag, Lunarion, 1993.

BílEK Jiř̌í, „Kulturně výchovná a zájmová umělecká činnost v ČSLA v letech 1948-1955 [Die kulturelle Erziehungs- und freizeitkünstlerische Tätigkeit in der CVA in den Jahren 1948-1955]“, in Studie k československým vojenským dějinám [Studien zur tschechoslowakischen Militärgeschichte], Prag, Vojenský historický ústav, 1987, S. 74-103.

BLAIVE Muriel, Une destalinisation manquée : Tchécoslovaquie 1956, Brüssel, Éditions Complexe, 2005.

HANKE Irma, „Kulturpolitik“, in Pipers Wörterbuch zur Politik, Bd. 4 (Sozialistische Systeme), ed. Klaus Ziemer, München, Piper, 1986, S. 233-238.

HAYDUK Ronald, „Progressives“, in International Encyclopedia of the Social Sciences, Bd. 6, 2. Aufl., Detroit, Mich., Macmillan, 2008, S. 536-538.

KAPLAN Karel, Kronika komunistického Československa: Alexej Čepička [Die Chronik der kommunistischen Tschechoslowakei: Alexej Čepička], Brno, Barrister \& Principal, 2011.

PERNES Jiří, POSPÍŠIL Jaroslav und LUKÁŠ Antonín, Alexej Čepička: Šedá eminence rudého režimu [Alexej Čepička: Die graue Eminenz des roten Regimes], Prag, Brána, 2008.

PIEKARSKI Stanisław, W cieniu muz [Im Schatten der Musen], Warschau, Dom Wojska Polskiego, 1999.

-, Z frontu na scenĘ: Tradycje Zespołu Artystycznego Wojska Polskiego [Aus der Front auf die Szene: Traditionen des Kunstensembles der Polnischen Armee], Warschau, Reprezentacyjny Zespół Artystyczny Wojska Polskiego, 2002.

RoGg Matthias, Armee des Volkes, Berlin, Ch. Links, 2008.

ŠACH Jan, „Písní, tancem a sportem bráníme mír“ [Mit Lied, Tanz und Sport verteidigen wir den Frieden], in J. Bílek, J. Láník und J. Šach, Československá armáda v prvním poválečném desetiletí květen 1945-květen 1955 [Die Tschechoslowakische Armee im ersten Nachkriegsjahrzehnt Mai 1945-Mai 1955], Prag, MO-AVIS, 2006, S. 174-187.

ŠMIDRKAL Václav, „O vzniku a úloze bratislavského Vojenského uměleckého souboru“ [Von der Entstehung und Rolle des Bratislavaer Armeekunstensembles], Vojenská história 14, Nr. 2, 2010, S. 60-74.

WEILER Bernd, „The Idea of Progress“, in The Blackwell Encyclopedia of Sociology, Bd. 7, ed. George Ritzer, Malden, Mass., Blackwell, 2007, S. 3669-3701.

WIATR Jerzy, Socjologia Wojska [Militärsoziologie], Warschau, Wydawnictwo MON, 1964. 
WYSZCZELSKI Lech, Oświata, propaganda, kultura w Wojsku Polskim w latach 1918-1945 [Aufklärung, Propaganda, Kultur in der Polnischen Armee in den Jahren 1918-1945], Warschau, Neriton, 2004. WoLF Friedrich, Kunst ist Waffe, Leipzig, Reclam, 1969.

\section{RÉSUMÉS}

Der Aufsatz beschäftigt sich mit der Kulturpolitik der sozialistischen Armee am Beispiel der Tschechoslowakischen Armee unter dem 1950-1956 amtierenden Minister für Nationale Verteidigung Alexej Čepička. Die kommunistische Idee des kulturellen Fortschritts setzte sich in dieser Etappe im Aufbau eines umfangreichen Netzes und sowohl hauptamtlicher wie auch ehrenamtlicher Kulturinstitutionen um, die von der Kommunistischen streng überwacht wurden. Der „cäsarische“ Regierungsstil des Ministers führte zu seiner Abberufung im April 1956 und auch zu einer breiten Kritik an seinen kulturpolitischen Maßnahmen.

This article deals with the cultural policy of the socialist army using the example of the Czechoslovak Army under the minister of national defense Alexej Čepička (tenure 1950-1956). The communist idea of progress in this period was translated into a construction of an extensive net of professional as well as amateur cultural institutions that were closely controlled by the communist party. The minister's "Caesarian" style of governing led to his dismissal in April 1956 and to a widespread criticism also about his decisions in the field of culture.

\section{INDEX}

Schlüsselwörter : 1950er, Kulturarbeit, Staatssozialismus, Tschechoslowakische Armee

Keywords : 1950s, cultural work, Czechoslovak Army, state socialism

\section{AUTEUR}

\section{VÁCLAV ŠMIDRKAL}

Karls-Universität Prag 